\title{
Evaluation of Wavefront Sensors Based on Etched Microlenses
}

\author{
R. E. Pierson, K. P. Bishop, E. Y. Chen
}

Applied Technology Associates, 1900 Randolph SE, Albuquerque, NM 87106, 505/846-6110, FAX: 505/768-1391

D. R. Neal

Sandia National Laboratories, P.0. Box 5800, Albuquerque, NM 87185-0601, 505/844-0854, FAX: 505/844-6098

\author{
L. McMackin
}

U. S. Air Force Phillips Laboratory, Kirtland AFB, NM 87117, 505/846-2046, FAX: 505/846-2045

\begin{abstract}
Binary etched microlenses enable fabrication of Shack-Hartmann wavefront sensors optimized for a particular application. We present a modeling and test approach to microlens optimization.
\end{abstract}

This work was suponried by the United States Department of Energy undor Coniract DE-ACก4-94AL 85000.

\section{DISCLAIMER}

This report was prepared as an account of work sponsored by an agency of the United States Government. Neither the United States Government nor any agency thereof, nor any of their employees, makes any warranty, express or implied, or assumes any legal liability or responsibility for the accuracy, completeness, or usefulness of any information, apparatus, product, or process disclosed, or represents that its use would not infringe privately owned rights. Reference herein to any specific commercial product, process, or service by trade name, trademark, manufacturer, or otherwise does not necessarily constitute or imply its endorsement, recommendation, or favoring by the United States Government or any agency thereof. The views and opinions of authors expressed herein do not necessarily state or reflect those of the United States Government or any agency thereof. 


\title{
Evaluation of Wavefront Sensors Based on Etched Microlenses
}

\author{
R. E. Pierson, K. B. Bishop, E. Y. Chen \\ Applied Technology Associates, 1900 Randolph SE, Albuquerque, NM 87106, 505/846-6110, FAX: 505/768-1391 \\ D. R. Neal \\ Sandia National Laboratories, P.O. Box 5800, Albuquerque, NM 87185-0601, 505/844-0854, FAX: 505/844-6098 \\ L. McMackin \\ U. S. Air Force Phillips Laboratory, Kirtland AFB, NM 87117, 505/846-2046, FAX: 505/846-2045
}

\begin{abstract}
1.0 Introduction
Since etched microlenses are produced by digital technology, it is inherently easy to fabricate optics customized for a particular application. However, optimization of wavefront sensors requires an ability to predict their complex behavior. In previous work, $^{1,2}$ we demonstrated binary microlens arrays for wavefront sensing in a visible wavelength tomographic imaging system. An eight-view tomographic system based on microlens array sensors is now operational and is described in a separate paper at this conference. ${ }^{3}$ The current paper addresses error budgeting and optimal design for wavefront sensors; it describes modeling and test procedures and illustrates one design approach.
\end{abstract}

\subsection{Etched Microlens Wavefront Sensors} The principle of Shack-Hartmann wavefront sensing is well documented. ${ }^{45}$ Figure 1 depicts a singleetched microlens with a detector array at its focal plane. The edge-to-edge wavefront optical path difference over the lens aperture is translated into a displacement of the focal spot at the detector array. A computer records the intensity profile at the detector array, calculates spot motion, and reconstructs the corresponding tilt at the microlens.

The microlenses are fabricated from a series of optical masks produced by commercial mask layout software. The mask pattern is transferred to the microlens substrate by coating the substrate with photo-resist, exposing through the mask with ultraviolet light, developing the substrate, and etching into the substrate using reactive-ion etching. The process is repeated with a sequence of masks, each etched to twice the depth of the preceding mask. For our application, we used four masks to produce 16 etched levels. Fewer etched levels cause power to be dissipated from the center of the focal spot to its tails; more levels require more etching and narrower etches. The manufacturing process restricts the narrowest possible etch width as well as the total sag across the

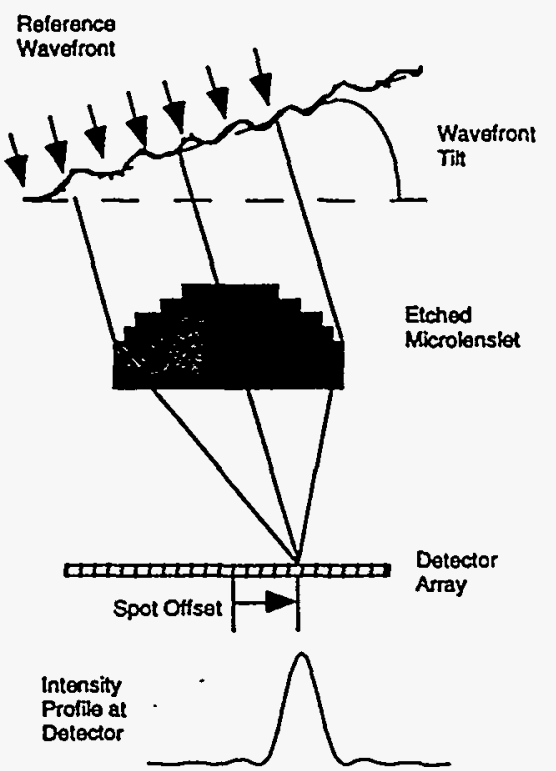

Figure 1. Binary microlenses from focal spots on a fast readout CCD array. Spot motion at the focal plane records wavefront tilt across the microlens.

microlens. For our application, the microlenses are on the order of $200-500$ microns with an f-number of 90. The resulting surfaces are approximately spherical with a total sag of one to two waves.

Figure 2 depicts a section of a microlens array in a Shack-Hartmann configuration. The motion of each microlens's focal plane spot is calculated and the corresponding tilt is integrated to reconstruct the impinging wavefront. The wavefront measuring ability of a sensor is often described in terms of range . and sensitivity where range is the maximum detectable tilt at each microlens and sensitivity is the minimum detectable tilt. For example, air flow simulations indicate that our application requires a sensitivity of about $3 \mu \mathrm{rad}$ at a range of $+1-100 \mu \mathrm{rad}$. However, one- and two-dimensional microlens arrays demonstrate more complex behavior than a single microlens. Attempts to achieve high sensitivity and 


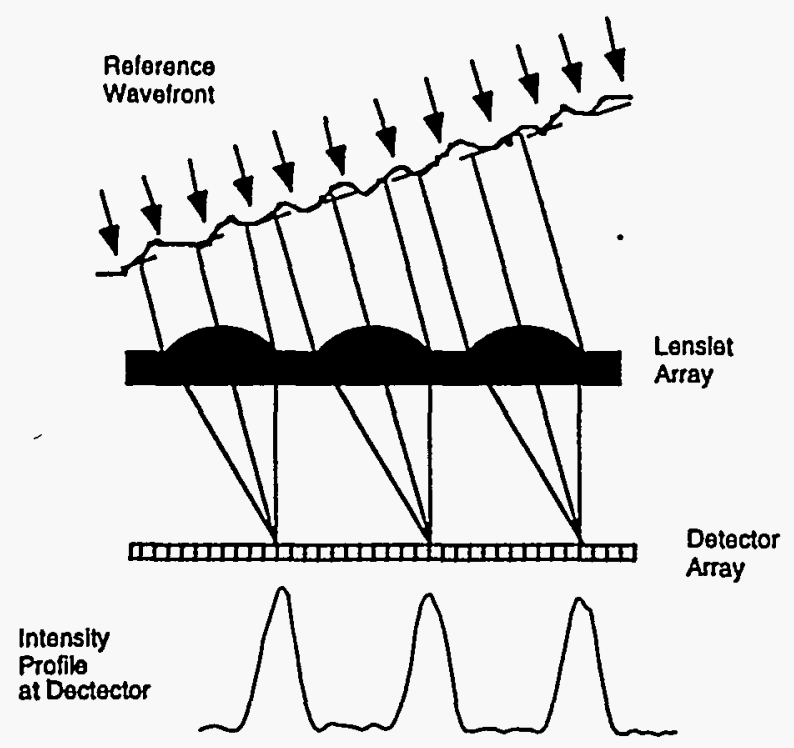

Figure 2. An array of etched microlenses samples wavefront tilts. Interference between focused spots increases with decreasing microlens spacing.

use coherent illumination may severely restrict the tilt measurement range due to interference between spots. The spot intensity profiles in Figure 2 for a microlens array show a markedly different form than the single spot in Figure 1 for a single microlens; this difference is due to destructive interference between spot side lobes.
3.0 Error Budget, Computer Models, and Laboratory Tests

Although the principle of the wavefront sensor system is simple, evaluating performance for a particular binary microlens design may be complicated. Figure 3 indicates this complexity by outlining the error budget for our wavefront sensor.

Random, short time-scale errors appear as noise in the spot location measurements from one time sample to the next. These errors include detector noise, electronic interference, and accuracy limits in the spot location algorithm. These errors can be mitigated by careful design but not removed. In contrast to short time-scale errors, long time-scale errors appear to accumulate with time. These errors are primarily due to changes in the reference wavefront that result in a "fixed pattern" in the wavefront tilt measurements. These errors can be mitigated by careful design, frequent calibration, and good experimental technique.

Deterministic errors result in consistent false measurements of tilt that vary from microlens to microlens over a whole array. These errors are due to physical system problems such as misalignment, positioning errors, or lens-to-lens differences. Deterministic errors can be removed by calibration against wavefronts of known tilt. In contrast, signaldependent errors are induced by the wavefront being

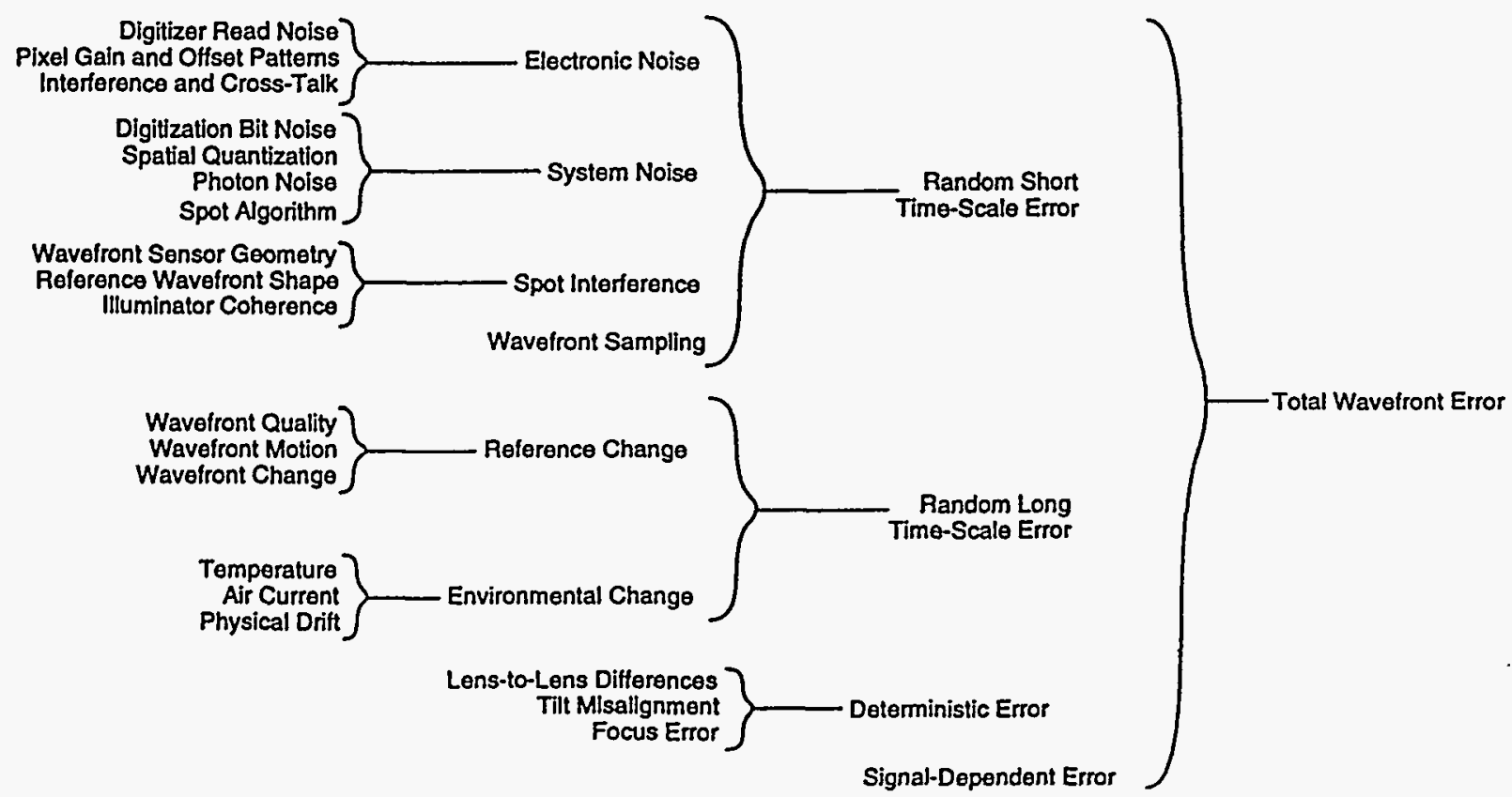

Figure 3. This error budget indicates both short and long time-scale noise sources in wavefront sensing. 
measured and are very difficult to correct. Large distortions may cause complex behaviors due to shifting of the reference wavefront or significant interference among focal plane spots.

Over the past year, we have quantified the error sources for our application. A full analysis of these results is beyond the scope of this paper. However, our method for evaluating the error budget is based on computer modeling anchored by laboratory tests.

The modeling system diagrammed in Figure 4 is implemented in software using Khoros and IDL image processing and visualization tools. The model performs a Monte-Carlo simulation of the wavefront sensing process. It creates a finely sampled reference wavefront, propagates it through the distortion, through the microlens array, and to the detector. The detected intensity is digitized, processed to locate focal plane spots, and converted back to wavefront tilts. The resulting tilts are compared to true tilts, or the integrated wavefront is compared to the known wavefront at the microlens array.

Figure 5 shows the laboratory test configuration. A collimated source is reflected off of a mirror at 45 degrees onto the microlens array. To calculate random short time-scale errors, all components of the system are kept fixed while focal plane measurements are repeated at $2.5 \mathrm{kHz}$ over a period of $4 \mathrm{~ms}$. The root-mean-square motion of all the focal plane spots over this short time period provides a measure of short time-scale error. In the same way, the system is kept fixed while data is collected over 30 minutes at intervals of 30 seconds. The root-mean-square difference between average focal plane spot locations at any time and the average positions at the beginning of the run is taken as the measure of accumulated long time-scale error. Deterministic errors are

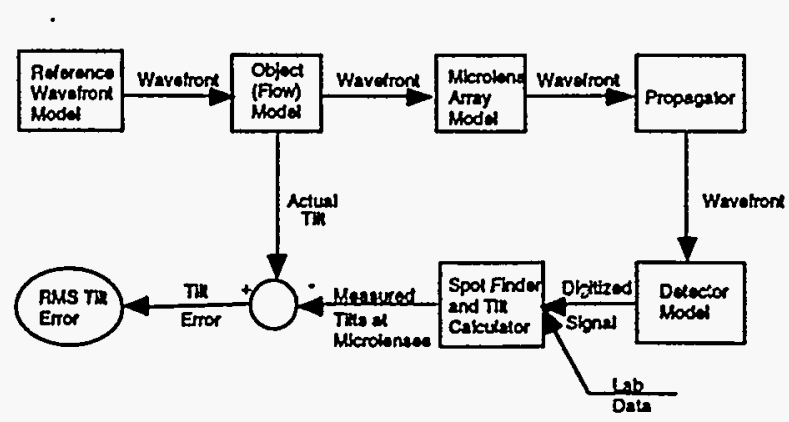

Figure 4. Computer simulations predict microlens array performance under realistic conditions of illumination and noise for expected flow conditions.

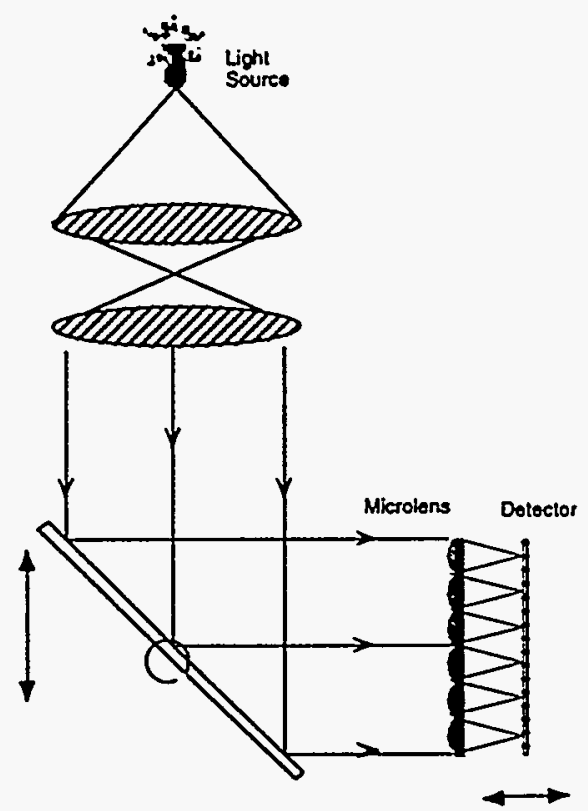

Figure 5. In this test configuration, the light source, microlens to detector distance, wavefront tilt, and wavefront position may all be controlled.

evaluated by introducing tilt at the mirror and measuring lens-to-lens response. If the exact amount of induced tilt is known, both relative and absolute errors may be corrected by appropriate scaling of the measured tilts. In contrast, signal-dependent errors are not directly measured. However, errors due to reference wavefront quality can be assessed by translating the reference wavefront across the sensor using the mirror. Changes in measured tilt, as the wavefront is translated, measure roughness and structure of the wavefront.

\subsection{A Design Approach}

The parameter space for microlens-array-based wavefront sensor design is very large. However, many of the parameters are fixed by the application. After limiting the degrees of freedom, the computer model described in the preceding section may be used to evaluate performance within the remaining design space. In addition, an error budget allows decoupling of weakly interrelated error sources. Random longterm error, random short-term error, deterministic error, and signal-dependent error may all be essentially independent over the range of possible designs being considered.

Figure 6 illustrates the method of optimal design for short-term error based on assumptions of two degrees of freedom: the number of microlenses per fixed length array and the focal length of these microlenses. 
To begin, we fix the number of microlenses and run performance simulations as a function of focal length. Our design parameter is root-mean-square tilt error due to random short time-scale errors. The resulting curve in Figure 6a shows optimal performance for focal lengths from $40-100 \mathrm{~mm}$. Performance is worse at shorter focal lengths because of decreased sensitivity and focal plane spot size. At longer focal lengths, performance is limited by interference among the focal plane spots. In Figure $6 \mathrm{~b}$, we determine the optimum performance for each microlens density; each point in this plot is the minimum of a curve similar to $6 a$ for a given number of microlenses per millimeter. Figure $6 \mathrm{~b}$ indicates that the tilt error increases with the number of microlenses due, primarily, to decreasing spot size. Figure $6 \mathrm{c}$ changes error metrics to add information about the wavefront to be reconstructed. Here the measure of error is the root-mean-square error between the reconstructed wavefront and the true wavefront measured as optical path difference. For a small number of microlenses, the wavefront error is large due to wavefront undersampling. For a large number of microlenses, the error is dominated by noise in the measured tilts. From this analysis, the optimum design point is near 2.8 lenses/mm, and the focal length at this design point happens to be $48 \mathrm{~mm}$.

In our application, our actual operating point is at $2.23 \mathrm{microlenses} / \mathrm{mm}$ and a focal length of $37.5 \mathrm{~mm}$. Our measured short time-scale root-mean-square tilt error at this configuration is $1.0 \mu \mathrm{rad}$, which matches the simulation prediction. Actual performance in our system is limited by long time-scale errors which have been measured at $3.0 \mu \mathrm{rad}$ over 30 minutes. It is our hope to present more details of the error budget and measured errors in a subsequent report.

\subsection{Conclusions}

Microlens-based designs are versatile and adaptable due to the ability to specify and fabricate microlenses optimized to a particular application. For ShackHartmann wavefront sensing, the complex system behavior can be simulated by computer and verified by simple laboratory tests. In addition, an errorbudget-based design approach allows discrimination of an optimal design for a particular wavefront sensing application.

This paper addressed only design issues for the wavefront sensor. A separate paper ${ }^{3}$ at this conference addresses design and operation of a full tomographic system based on microlens arrays.

\subsection{References}

1. McMackin, L., Masson, B., Clark, N., Bishop, K., Pierson, R., Chen, E., "Hartmann Wavefront Sensor Studies of Dynamic Organized Structure in Flowfields," AlAA Journal, Vol. 33. No. 11, pp. 2158-2164, November 1995.

2. Bishop, K., Chen, E., McMackin, L., Neal, D., Pierson, R. "One-Dimensional Wavefront Sensor Development for Tomographic Flow Measurements," SPIE, August 1995.

3. Bishop, K., Chen, E., McMackin, L., Pierson, R.. "A Multiview Fast Optical Tomography System Using Microlens Arrays," Optical Society of America Conference on Diffractive Optics and Micro-Optics, Boston, Massachusetts, April 29 - May 3, 1996.

4. Schack, R. V., "Direct Phase Sensing Interferometer,". Journal of the Optical Society of America, Vol. 61, No. 5 . p. $655,1971$.

5. Schack, R. V. and Platt, B. C., "Production and Use of a Lenticular Hartmann Screen," Joumal of the Optical Society of America, Vol. 61, No. 5, p. 656, 1971.

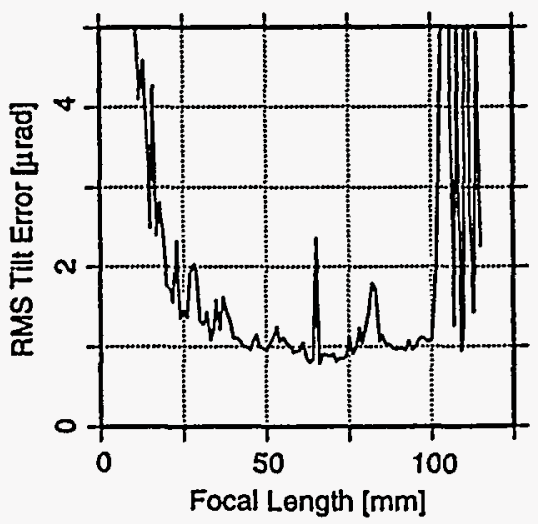

a) Tilt Error versus Focal Length

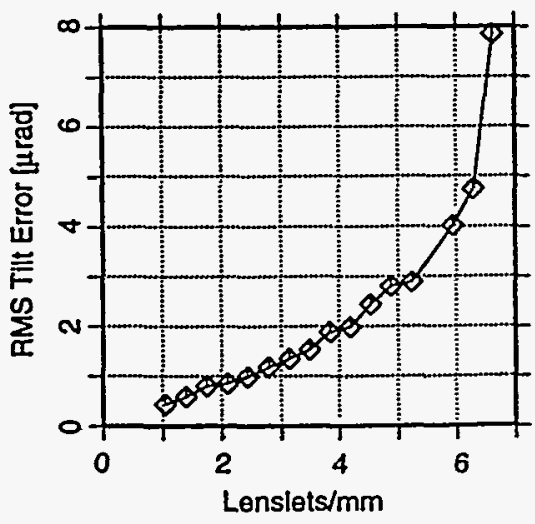

b) Tilt Error versus Number of Lenslets

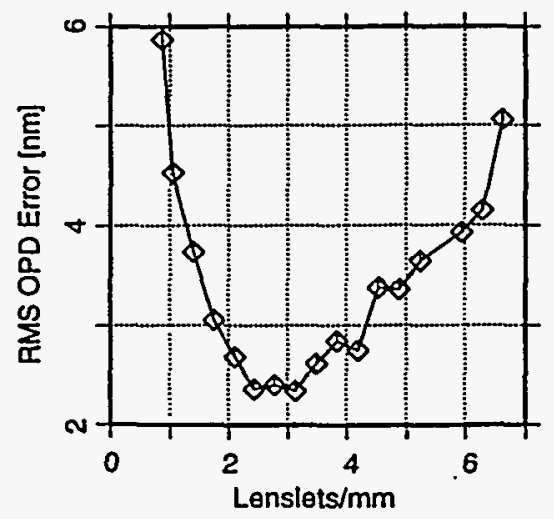

c) Wavefront Error versus Number of Lenslets

Figure 6. Design optimization is based on predicted performance evaluated over the free parameter space -- in this case f-number and microlens density. 